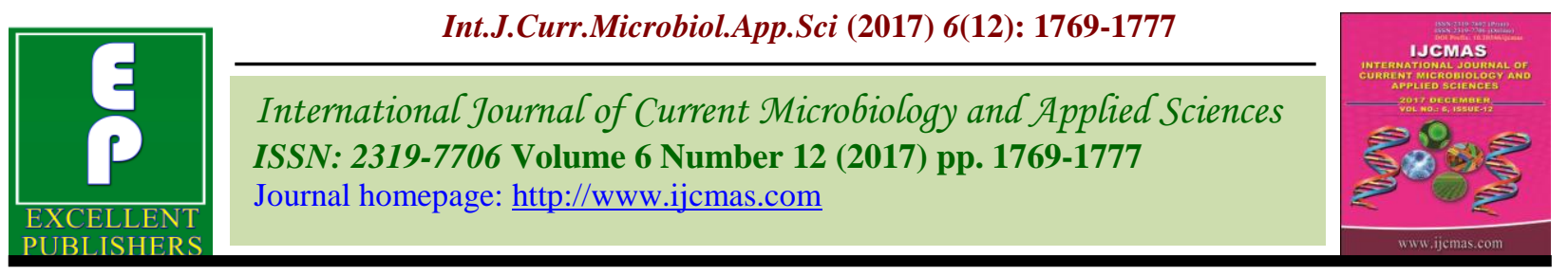

Original Research Article

https://doi.org/10.20546/ijcmas.2017.612.200

\title{
Extent of Use of different Agriculture Information Sources and Channels by the Aonla Growers
}

\author{
S. Choudhary* and I.M. Khan \\ Department of extension education, SKN, COA, Jobner, Rajasthan, India \\ *Corresponding author
}

\begin{tabular}{|l|l}
\hline \multicolumn{1}{l}{ A B S T R A C T } \\
\cline { 2 - 3 } \multicolumn{1}{l|}{$\begin{array}{l}\text { Keywords } \\
\begin{array}{l}\text { Agriculture } \\
\text { information, } \\
\text { Sources and } \\
\text { Channels. }\end{array}\end{array}$} & $\begin{array}{l}\text { India has one of the largest and most complex public systems for } \\
\text { generation, testing and transfer of agricultural information. It is the } \\
\text { information behaviour of the farmers, which can promote and spread the } \\
\text { results obtained in the laboratories for their better utilization in farming } \\
\text { community. Aonla growers information management from different sources } \\
\text { and channels of agriculture information which have brought the aonla } \\
\text { growers and scientists close to understand the suitability of the } \\
\text { technologies. The Semi-Arid Eastern Plains Zone (IIIa) of Rajasthan has } \\
\text { highest area and production under aonla cultivation. There are so many } \\
\text { agricultural institutions, which are engaged in the research on aonla } \\
\text { growers problems and transfer of technology to the aonla growers. } \\
\text { Available Online: } \\
\text { 10 December 2017 }\end{array}$ \\
\hline \hline
\end{tabular}

\section{Introduction}

An improvement and strengthening of agricultural infrastructure needed to all the levels of supply chain. Shrinking extension is another component of infrastructure that needs attention. After the green revolution in the mid-sixties there has been no major technological innovation, which could give a fresh impetus to agricultural productivity, insufficient extension services and poor access to information further widen the gap in the adoption of technology and lead to poor productivity levels. A push towards higher productivity will require information based, decision making agricultural system. This is often described as the next great evolutionary step in agricultural. Today's farmers desire not only the meals for their families from their hard sweat but also surplus production which can sold in the market to get sufficient money to fulfill the daily requirements. According to economic reforms in the country each and every sector has changed its strategies in view of global competition.

Among the various sources of information mass media is an important one which consists of newspapers, magazines, traditional media, radio, TV and information technology which have proven to be the most powerful opinion makers in this information technology era. They cover more people in less time with minimum cost. This strength of mass media is 
of great help to extension workers in providing cost effective and efficient services to the farmers. Computers are the greatest discovery in the recent times. Internet has made the world into a global village and provides the channel for exchange of information quickly. Unfortunately the usage of information technology as a powerful medium in the field of agriculture is yet to take place effectively.

\section{Materials and Methods}

The extent of use of different sources and channels of agriculture information was measured by using the scale developed by Singh (2002) with little modification as suggested by the experts on a four point continuum namely 'most often', 'often', 'some time' and 'never' with a scale of 3, 2, 1 and 0 respectively.

For example if out of 10 sources and channels, 3 were perceived as the 'most often', 2 were perceived as 'often', 3 were perceived as 'some time' and 2 were perceived as 'never', by a particular respondent, then score of extent of use of that particular respondent will be $(3 \times 3)+(4 \times 2)$ $+(3 \times 1)+(2 \times 0)=20$

\section{Results and Discussion}

The extent of use of different agriculture information sources and channels for improved aonla cultivation practices as perceived by the respondents in the study area. All the possible extent, faced by the respondents were grouped into four major categories viz., personal localite sources, personal cosmopolite sources, personal cosmopolite channels and impersonal cosmopolite channels. The data regarding the extent of use by different categories of aonla growing farmers and farm women has been presented into following sub heads.
Distribution of aonla growers according to their different levels of use of agriculture information sources and channels

Extent of use of different personal localite sources of agriculture information by the aonla growing farmers and farm women

Extent of use of different personal cosmopolite sources of information by the aonla growing farmers and farm women

Extent of use of different personal cosmopolite channels of information by the aonla growing farmers and farm women

Extent of use of different impersonal cosmopolite channels of information by the aonla growing farmers and farm women

\section{Distribution of aonla growers according to their different levels of use of agriculture information sources and channels}

The extent of use of particular aonla growing farmers and farm women was calculated by summing the scores obtained by that particular farmer in all the four components of extent of use which are personal localite sources, personal cosmopolite sources, personal cosmopolite channels and impersonal cosmopolite channels. Then the aonla growing farmers and farm women were categorized into five different levels of extent of use namely, very low ( $0-20$ per cent), low (20-40 per cent), medium (40-60 per cent), high (60-80 per cent) and very high (80-100 per cent). Further the $X^{2}$ value between the frequencies of aonla growing farmers and farm women in different levels was calculated to find out the significance in the agreement between the aonla growing farmers and farm women.

The data presented in Table 1 indicates that majority of the aonla growing farmers and 
farm women (56.00 per cent and 52.00 per cent respectively) were having high extent of use, whereas 35.00 per cent aonla growing farmers and 38.00 per cent farm women were having medium extent of use. Only 2.50 per cent aonla growing farmers and 0.83 per cent farm women were having very high extent of use. None of the aonla growing farmers and farm women were having very low extent of use of agriculture information sources and channels.

The data in Table 1 indicates that majority of aonla growing farmers and farm women (60.00 per cent and 55.00 per cent respectively) were having high extent of use in Jaipur district, whereas 47.50 per cent aonla growing farmers and 45.00 per cent farm women were having high extent of use in Ajmer district. In Jaipur district 32.50 per cent aonla growing farmers and 35.00 per cent farm women were having medium extent of use of agriculture information sources and channels, however 40.00 per cent aonla growing farmers and 42.50 per cent farm women were having medium extent of use of agriculture information sources and channels in Ajmer district. Only 2.50 per cent aonla growing farmers and 1.25 per cent farm women were having very high extent of use in Jaipur district, whereas in Ajmer district only 2.50 per cent aonla growing farmers and none of the farm women were having very high extent of use. None of the aonla growing farmers and farm women in both districts were having very low extent of use of agriculture information sources and channels.

The calculated value of chi-square $\left(\chi^{2}\right)$ test between the scores of aonla growing farmers and farm women was found to 96.02 , which is more than its tabulated value (9.49) at 5 per cent level of significance. Hence the null hypothesis was rejected and alternate hypothesis was accepted. This leads to the conclusion that there is a highly significant agreement between the aonla growing farmers and farm women with regard to their extent of use of agriculture information sources and channels.

\section{Extent of use of different personal localite sources of information by the aonla growing farmers and farm women}

The data related with extent of use of different personal localite sources of agriculture information of the aonla growing farmers and farm women incorporated in Table 2 shows that calculated Wilcoxen ' $Z$ ' value for the extent of use of personal localite sources viz. 'progressive famers', 'friends', 'neighbours', 'relatives', 'agriculture graduates', 'family member' and 'opinion leaders' were more than the tabulated value at 5 per cent level of significance. Hence, the null hypothesis was rejected and alternate hypothesis was accepted, which leads to the conclusion that there is a significant difference between aonla growing farmers and farm women with respect to the extent of use of these personal localite sources of information. Whereas the calculated Wilcoxen ' $Z$ ' value for the 'panchayat members' was less than the tabulated value at 5 per cent level of significance. Hence, the null hypothesis was accepted and alternate hypothesis was rejected, which leads to the conclusion that there is no significant difference between aonla growing farmers and farm women with respect to the extent of use of these personal localite sources of information.

The data presented in Table 2 indicates that for aonla growing farmers and farm women "progressive farmer" (MPS 87.08 and 85.00 respectively) and "family members" (MPS 78.63 and 77.71 respectively) were the major personal localite sources of agriculture information used by majority of the aonla growers and accorded first and second ranks 
respectively. It also revealed that for aonla growing farmers and farm women "Agriculture graduates" (MPS 56.67 and 54.59 respectively) was the least preferred information sources.

In case of aonla growing farmers and farm women in Jaipur district "progressive farmers" (MPS 78.33 and 80.42 respectively) was the major personal localite sources of agriculture information used by majority of the aonla growers and accorded first rank, however, for aonla growing farmers "opinion leaders" (MPS 82.08) and for farm women "family members" (MPS79.58) were the major personal localite sources of agriculture information used by majority of the aonla growers and accorded second rank. It was also revealed that for aonla growing farmers and farm women "Agriculture graduates" (MPS 52.50 and 51.67 respectively) was the least preferred information sources.

Whereas, for aonla growing farmers and farm women "progressive farmer" (MPS 85.83 and 86.67 respectively) and "family members" (MPS 81.33 and 75.83 respectively) in Ajmer district were the major personal localite sources of agriculture information used by majority of the aonla growers and accorded first and second ranks respectively. It was also revealed that for aonla growing farmers and farm women "Agriculture graduates" (MPS 57.50 and 56.67 respectively) was the least preferred information source.

The conclusion can be drawn on the basis of data that "progressive farmers" and "family members" were the major personal localite sources of agriculture information used by majority of the respondents. Therefore, it is suggested that efforts should be made to equip the "progressive farmers" and "family members" with latest knowledge to communicate agriculture information more effectively and efficiently in the study area.
Extent of use of different personal cosmopolite sources of agriculture information by the aonla growing farmers and farm women

The data related with extent of use of different personal cosmopolite sources of agriculture information of the aonla growing farmers and farm women incorporated in Table 3 shows that calculated Wilcoxen ' $Z$ ' value for the extent of use of personal cosmopolite sources viz. 'agriculture supervisor', 'A.R.S/Master trainers of agriculture', 'NGO's personnel', 'agriculture officer', 'Asstt. agriculture officer', 'private agencies', 'research station', 'plant clinic/polyclinic center', 'co-operative officials', 'panchayat officials', 'Deputy director of agriculture', 'Asstt. director of agriculture and K.V.K. officials' were more than the tabulated value at 5 per cent level of significance. Hence, the null hypothesis was rejected and alternate hypothesis was accepted, which leads to the conclusion that there is a significant difference between aonla growing farmers and farm women with respect to these personal cosmopolite sources of information. Whereas the calculated Wilcoxen ' $Z$ ' value for the extent of use of personal cosmopolite sources viz. 'salesmen and dealers' and 'A.T.I.C' were less than the tabulated value at 5 per cent level of significance. Hence, the null hypothesis was accepted and alternate hypothesis was rejected, which leads to the conclusion that there is no significant difference between aonla growing farmers and farm women with respect to the extent of use of these personal cosmopolite sources of information.

The data presented in Table 3 indicates that for aonla growing farmers and farm women "agriculture supervisor" (MPS 88.34 and 87.50 respectively) and "salesman and dealers" (MPS 84.58 and 78.71 respectively) were the major personal cosmopolite sources 
of agriculture information used by majority of the aonla growers and accorded first and second ranks respectively. It was also revealed that for aonla growing farmers and farm women "plant clinic/polyclinic center" (MPS 45.83) and for farm women "deputy director of agriculture" (MPS 47.09) were the least preferred information sources.

In case of aonla growing farmers and farm women "agriculture supervisor" (MPS 87.50 and 86.67 respectively) and "salesman and dealers" (MPS 83.33 and 77.08 respectively) in Jaipur district were the major personal cosmopolite sources of agriculture information used by majority of the aonla growers and accorded first and second ranks respectively. It was also revealed that for aonla growing farmers "plant clinic/polyclinic center" (MPS 43.33) and for farm women "Assistant director of agriculture" (MPS 45.42) were the least preferred information sources. However, for the aonla growing farmers and farm women in Ajmer district "agriculture supervisor" (MPS 89.17 and 88.33 respectively) and "salesman and dealers" (MPS 85.83 and 80.33 respectively) were the major personal cosmopolite sources of agriculture information used by majority of the aonla growers and accorded first and second ranks respectively. It also revealed that for aonla growing farmers "plant clinic/polyclinic center" (MPS 48.33) and for farm women "deputy director of agriculture" (MPS 45.00) were the least preferred information source as perceived by the.

Table.1 Distribution of aonla growing farmers and farm women according to their different levels of use of agriculture information management

\begin{tabular}{|c|c|c|c|c|c|c|c|}
\hline \multirow{2}{*}{$\begin{array}{l}\text { S. } \\
\text { No. }\end{array}$} & \multirow{2}{*}{$\begin{array}{l}\text { Levels use of agriculture } \\
\text { information management }\end{array}$} & \multicolumn{2}{|c|}{$\begin{array}{c}\text { Jaipur district } \\
(n=160)\end{array}$} & \multicolumn{2}{|c|}{$\begin{array}{c}\text { Ajmer district } \\
(n=80)\end{array}$} & \multicolumn{2}{|c|}{$\begin{array}{c}\text { Total respondents } \\
(\mathrm{N}=\mathbf{2 4 0})\end{array}$} \\
\hline & & $\begin{array}{c}\text { Farmers } \\
(\mathbf{n}=\mathbf{8 0})\end{array}$ & $\begin{array}{c}\text { Farm women } \\
(\mathrm{n}=\mathbf{8 0})\end{array}$ & Farmers $(n=40)$ & $\begin{array}{c}\text { Farm women } \\
(n=40)\end{array}$ & $\begin{array}{c}\text { Farmers } \\
(n=120)\end{array}$ & $\begin{array}{c}\text { Farm women } \\
(n=120)\end{array}$ \\
\hline 1. & Very Low (0-20 per cent) & $\begin{array}{c}0 \\
(0.00)\end{array}$ & $\begin{array}{c}0 \\
(0.00) \\
\end{array}$ & $\begin{array}{c}0 \\
(0.00) \\
\end{array}$ & $\begin{array}{c}0 \\
(0.00)\end{array}$ & $\begin{array}{c}0 \\
(0.00)\end{array}$ & $\begin{array}{c}0 \\
(0.00)\end{array}$ \\
\hline 2. & Low (20-40 per cent) & $\begin{array}{c}4 \\
(5.00) \\
\end{array}$ & $\begin{array}{c}7 \\
(8.75) \\
\end{array}$ & $\begin{array}{c}4 \\
(10.00) \\
\end{array}$ & $\begin{array}{c}5 \\
(12.50) \\
\end{array}$ & $\begin{array}{c}8 \\
(6.70) \\
\end{array}$ & $\begin{array}{c}12 \\
(10.00)\end{array}$ \\
\hline 3. & Medium (40-60 per cent) & $\begin{array}{c}26 \\
(32.50) \\
\end{array}$ & $\begin{array}{c}28 \\
(35.00) \\
\end{array}$ & $\begin{array}{c}16 \\
(40.00) \\
\end{array}$ & $\begin{array}{c}17 \\
(42.50) \\
\end{array}$ & $\begin{array}{c}42 \\
(35.00) \\
\end{array}$ & $\begin{array}{c}45 \\
(38.00) \\
\end{array}$ \\
\hline 4 & High $(60-80$ per cent $)$ & $\begin{array}{c}48 \\
(60.00) \\
\end{array}$ & $\begin{array}{c}44 \\
(55.00) \\
\end{array}$ & $\begin{array}{c}19 \\
(47.50) \\
\end{array}$ & $\begin{array}{c}18 \\
(45.00) \\
\end{array}$ & $\begin{array}{c}67 \\
(56.00) \\
\end{array}$ & $\begin{array}{c}62 \\
(52.00) \\
\end{array}$ \\
\hline 5 & Very high ( $80-100$ per cent) & $\begin{array}{c}2 \\
(2.50)\end{array}$ & $\begin{array}{c}1 \\
(1.25)\end{array}$ & $\begin{array}{c}1 \\
(2.50)\end{array}$ & $\begin{array}{c}0 \\
(0.00)\end{array}$ & $\begin{array}{c}3 \\
(2.50)\end{array}$ & $\begin{array}{c}1 \\
(0.83)\end{array}$ \\
\hline & Total & $\begin{array}{c}\mathbf{8 0} \\
(100.00)\end{array}$ & $\begin{array}{c}\mathbf{8 0} \\
(\mathbf{1 0 0 . 0 0 )}\end{array}$ & $\begin{array}{c}40 \\
(10.00)\end{array}$ & $\begin{array}{c}40 \\
(10.00)\end{array}$ & $\begin{array}{c}120 \\
(100.00)\end{array}$ & $\begin{array}{c}120 \\
(100.00)\end{array}$ \\
\hline
\end{tabular}

$\mathrm{X}^{2}=96.02$ d.f. $=4$ Figures in parentheses indicate percentage

Table.2 Extent of use of different personal localite sources of agriculture information by the aonla growers

\begin{tabular}{|c|c|c|c|c|c|c|c|c|c|c|c|c|c|c|}
\hline \multirow{3}{*}{$\begin{array}{l}\text { S. } \\
\text { No. }\end{array}$} & \multirow{3}{*}{$\begin{array}{l}\text { Personal Localite } \\
\text { Sources }\end{array}$} & \multicolumn{4}{|c|}{$\begin{array}{l}\text { Jaipur district } \\
(\mathrm{N}=160)\end{array}$} & \multicolumn{4}{|c|}{$\begin{array}{l}\text { Ajmer district } \\
(\mathrm{N}=80)\end{array}$} & \multicolumn{4}{|c|}{$\begin{array}{c}\text { Total respondents } \\
(\mathrm{N}=\mathbf{2 4 0})\end{array}$} & \multirow[t]{3}{*}{$\begin{array}{l}\text { Wilcoxen } \\
\text { (z value) }\end{array}$} \\
\hline & & \multicolumn{2}{|c|}{ Farmers $(\mathbf{n}=\mathbf{8 0})$} & \multicolumn{2}{|c|}{$\begin{array}{c}\text { Farm women } \\
(n=80)\end{array}$} & \multicolumn{2}{|c|}{ Farmers $(n=40)$} & \multicolumn{2}{|c|}{$\begin{array}{c}\text { Farm women } \\
(n=40)\end{array}$} & \multicolumn{2}{|c|}{$\begin{array}{c}\text { Farmers } \\
(n=120)\end{array}$} & \multicolumn{2}{|c|}{$\begin{array}{c}\text { Farm women } \\
(\mathbf{n}=\mathbf{1 2 0})\end{array}$} & \\
\hline & & MPS & $\begin{array}{l}\text { Ra } \\
\text { nk }\end{array}$ & MPS & $\begin{array}{c}\text { Ran } \\
\mathbf{k}\end{array}$ & MPS & $\begin{array}{c}\text { Ran } \\
\mathbf{k}\end{array}$ & MPS & Rank & MPS & $\begin{array}{c}\text { Ran } \\
\mathbf{k}\end{array}$ & MPS & Rank & \\
\hline 1. & Progressive famers & 88.33 & I & 83.33 & I & 85.83 & I & 86.67 & I & 87.08 & I & 85.00 & I & $3.79 *$ \\
\hline 2. & Friends & 65.42 & $\mathrm{~V}$ & 64.58 & VI & 72.5 & IV & 70.83 & $\mathrm{~V}$ & 68.96 & IV & 67.71 & $\mathrm{~V}$ & $3.97 *$ \\
\hline 3. & Neighbours & 58.83 & VII & 70.42 & IV & 69.67 & $\mathrm{~V}$ & 74.17 & III & 64.25 & VI & 72.30 & IV & $2.95 *$ \\
\hline 4 & Relatives & 61.67 & VI & 73.75 & III & 65.83 & VI & 72.5 & IV & 63.75 & VII & 76.04 & III & $2.56 *$ \\
\hline 5 & $\begin{array}{l}\text { Agriculture } \\
\text { graduates }\end{array}$ & 52.5 & VIII & 51.67 & VIII & 60.83 & VIII & 57.5 & VIII & 56.67 & VIII & 54.59 & VIII & $3.17 *$ \\
\hline 6 & Panchayat members & 69.58 & IV & 60.83 & VII & 62.50 & VII & 62.5 & $\mathrm{~V}$ & 66.04 & $\mathrm{~V}$ & 61.67 & VII & 1.76 \\
\hline 7 & Family member & 75.92 & III & 79.58 & III & 81.33 & II & 75.83 & II & 78.63 & II & 77.71 & II & $4.18 *$ \\
\hline 8 & Opinion leaders & 82.08 & II & 66.25 & $\mathrm{~V}$ & 74.17 & III & 68.33 & VI & 78.13 & III & 67.29 & VI & $3.26 *$ \\
\hline
\end{tabular}

$*$ = significant at 5 per cent level of significance 
Table.3 Extent of use of different personal cosmopolite sources of agriculture information by the aonla growers

\begin{tabular}{|c|c|c|c|c|c|c|c|c|c|c|c|c|c|c|}
\hline \multirow{3}{*}{$\begin{array}{l}\text { S. } \\
\text { No. }\end{array}$} & \multirow{3}{*}{ Personal Cosmopolite Sources } & \multicolumn{4}{|c|}{ Jaipur district (N=160) } & \multicolumn{4}{|c|}{ Ajmer district $(\mathrm{N}=\mathbf{8 0})$} & \multicolumn{4}{|c|}{$\begin{array}{c}\text { Total respondents } \\
(\mathrm{N}=\mathbf{2 4 0})\end{array}$} & \multirow[t]{3}{*}{$\begin{array}{r}\text { Wilcoxen } \\
\text { ( } \mathrm{Z} \text { value) }\end{array}$} \\
\hline & & \multicolumn{2}{|c|}{$\begin{array}{c}\text { Farmers } \\
(n=80)\end{array}$} & \multicolumn{2}{|c|}{$\begin{array}{c}\text { Farm women } \\
(n=80)\end{array}$} & \multicolumn{2}{|c|}{$\begin{array}{c}\text { Farmers } \\
(n=40)\end{array}$} & \multicolumn{2}{|c|}{$\begin{array}{c}\text { Farm women } \\
(n=40)\end{array}$} & \multicolumn{2}{|c|}{$\begin{array}{c}\text { Farmers } \\
(\mathbf{n}=120)\end{array}$} & \multicolumn{2}{|c|}{$\begin{array}{c}\text { Farm women } \\
(n=120)\end{array}$} & \\
\hline & & MPS & $\begin{array}{l}\text { Ra } \\
\text { nk }\end{array}$ & MPS & Rank & MPS & Rank & MPS & $\begin{array}{l}\text { Ra } \\
\text { nk }\end{array}$ & MPS & $\begin{array}{l}\text { Ra } \\
\text { nk }\end{array}$ & MPS & Rank & \\
\hline 1. & Agriculture supervisor & 87.50 & $\mathrm{I}$ & 86.67 & I & 89.17 & I & 88.33 & I & 88.34 & I & 87.50 & I & $4.50 *$ \\
\hline 2. & $\begin{array}{l}\text { A.R.S/Master trainers of } \\
\text { agriculture }\end{array}$ & 52.08 & XI & 56.25 & $\mathrm{X}$ & 53.33 & XII & 57.50 & $\mathrm{X}$ & 52.71 & XII & 56.88 & $\mathrm{X}$ & $2.32 *$ \\
\hline 3. & Salesmen and dealers & 77.08 & II & 83.33 & II & 85.83 & II & 80.33 & II & 81.46 & II & 81.83 & II & 1.38 \\
\hline 4. & NGO's personnel & 60.80 & VII & 70.83 & IV & 69.17 & VI & 73.33 & IV & 64.99 & VII & 72.08 & III & $3.25^{*}$ \\
\hline 5. & Agriculture officer & 67.90 & $\mathrm{~V}$ & 63.33 & VII & 74.20 & IV & 78.33 & III & 71.05 & $\mathrm{~V}$ & 70.83 & IV & $3.14 *$ \\
\hline 6. & Asstt. agriculture officer & 70.83 & IV & 61.25 & $\begin{array}{c}\text { VII } \\
\text { I }\end{array}$ & 72.50 & $\mathrm{~V}$ & 60.83 & $\begin{array}{c}\text { VII } \\
\text { I }\end{array}$ & 71.67 & IV & 61.04 & IX & $3.06^{*}$ \\
\hline 7. & Private agencies & 74.58 & III & 59.67 & IX & 80.83 & III & 64.20 & VII & 77.71 & III & 61.94 & VIII & $2.69 *$ \\
\hline 8. & Research station & 50.42 & XII & 52.92 & XII & 55.83 & $\mathrm{XI}$ & 52.50 & XII & 53.13 & $\mathrm{XI}$ & 52.71 & XIII & $2.87 *$ \\
\hline 9. & Plant clinic/polyclinic center & 43.33 & $\mathrm{XV}$ & 54.60 & XI & 48.33 & $\begin{array}{c}\mathrm{XI} \\
\mathrm{V}\end{array}$ & 51.17 & $\begin{array}{c}\text { XII } \\
\text { I }\end{array}$ & 45.83 & $\mathrm{XV}$ & 52.89 & XI & $2.81 *$ \\
\hline 10 & A.T.I.C. & 57.92 & $\begin{array}{c}\text { VII } \\
\text { I } \\
\end{array}$ & 68.75 & $\mathrm{~V}$ & 65.00 & $\begin{array}{c}\text { VII } \\
\text { I }\end{array}$ & 70.00 & $\mathrm{~V}$ & 61.46 & $\begin{array}{c}\text { VII } \\
\text { I } \\
\end{array}$ & 69.38 & $\mathrm{~V}$ & 1.66 \\
\hline 11. & Co-operative officials & 55.42 & IX & 66.25 & VI & 59.17 & IX & 66.67 & VI & 57.30 & IX & 66.46 & VI & $2.97 *$ \\
\hline 12. & Panchayat officials & 52.50 & $\mathrm{X}$ & 51.25 & $\begin{array}{c}\text { XII } \\
\text { I }\end{array}$ & 58.25 & $\mathrm{X}$ & 54.20 & $\mathrm{XI}$ & 55.38 & $\mathrm{X}$ & 52.73 & XII & $1.97^{*}$ \\
\hline 13. & Deputy director of agriculture & 48.33 & $\begin{array}{c}\text { XII } \\
\text { I }\end{array}$ & 49.17 & $\begin{array}{c}\mathrm{XI} \\
\mathrm{V}\end{array}$ & 50.83 & $\begin{array}{c}\text { XII } \\
\text { I }\end{array}$ & 45.00 & $\mathrm{XV}$ & 49.58 & $\begin{array}{c}\text { XII } \\
\text { I }\end{array}$ & 47.09 & $\mathrm{XV}$ & $2.61^{*}$ \\
\hline 14. & Asstt. director of agriculture & 45.42 & $\begin{array}{c}\mathrm{XI} \\
\mathrm{V} \\
\end{array}$ & 42.50 & $\mathrm{XV}$ & 47.50 & $\mathrm{XV}$ & 58.33 & $\begin{array}{l}\mathrm{XI} \\
\mathrm{V}\end{array}$ & 46.46 & $\begin{array}{c}\mathrm{XI} \\
\mathrm{V} \\
\end{array}$ & 50.42 & XIV & $3.13^{*}$ \\
\hline 15. & K.V.K. officials & 64.58 & VI & 72.50 & III & 66.67 & VII & 59.17 & IX & 65.63 & VI & 65.84 & VII & $2.55^{*}$ \\
\hline
\end{tabular}

$*$ = significant at 5 per cent level of significance

Table.4 Extent of use of different personal cosmopolite channels of agriculture information by the aonla growers $\mathrm{N}=240$

\begin{tabular}{|c|c|c|c|c|c|c|c|c|c|c|c|c|c|c|}
\hline \multirow{3}{*}{$\begin{array}{l}\text { S. } \\
\mathbf{N} \\
\text { o. }\end{array}$} & \multirow[t]{3}{*}{ Personal Cosmopolite Channels } & \multicolumn{4}{|c|}{ Jaipur district ( $\mathrm{N}=160)$} & \multicolumn{4}{|c|}{ Ajmer district $(\mathrm{N}=\mathbf{8 0})$} & \multicolumn{4}{|c|}{$\begin{array}{c}\text { Total respondents } \\
(\mathrm{N}=\mathbf{2 4 0})\end{array}$} & \multirow[t]{3}{*}{$\begin{array}{l}\text { Wilcoxen } \\
\text { (Z value) }\end{array}$} \\
\hline & & \multicolumn{2}{|c|}{$\begin{array}{c}\text { Farmers } \\
(\mathbf{n}=80)\end{array}$} & \multicolumn{2}{|c|}{$\begin{array}{c}\text { Farm women } \\
(\mathbf{n}=80)\end{array}$} & \multicolumn{2}{|c|}{$\begin{array}{c}\text { Farmers } \\
(\mathrm{n}=40)\end{array}$} & \multicolumn{2}{|c|}{$\begin{array}{c}\text { Farm women } \\
(\mathrm{n}=40)\end{array}$} & \multicolumn{2}{|c|}{$\begin{array}{c}\text { Farmers } \\
(n=120)\end{array}$} & \multicolumn{2}{|c|}{$\begin{array}{c}\text { Farm women } \\
(\mathbf{n}=120)\end{array}$} & \\
\hline & & MPS & $\begin{array}{l}\text { Ra } \\
\text { nk }\end{array}$ & MPS & $\begin{array}{l}\text { Ra } \\
\text { nk }\end{array}$ & MPS & $\begin{array}{l}\text { Ra } \\
\text { nk }\end{array}$ & MPS & $\begin{array}{l}\text { Ra } \\
\text { nk }\end{array}$ & MPS & $\begin{array}{l}\text { Ra } \\
\text { nk }\end{array}$ & MPS & $\begin{array}{l}\text { Ra } \\
\text { nk }\end{array}$ & \\
\hline 1. & Training & 72.08 & VI & 78.75 & III & 67.80 & VII & 75.00 & $\mathrm{~V}$ & 69.94 & VI & 76.88 & III & $2.76^{*}$ \\
\hline 2. & Farmers fair (Kisan Mela) & 85.83 & I & 84.17 & I & 84.20 & I & 83.33 & I & 85.02 & I & 83.75 & I & $3.28^{*}$ \\
\hline 3. & Result demonstration & 76.83 & $\mathrm{~V}$ & 72.08 & $\mathrm{~V}$ & 72.50 & $\mathrm{~V}$ & 76.70 & IV & 74.67 & IV & 74.39 & IV & $3.83^{*}$ \\
\hline 4 & Method demonstration & 75.42 & VI & 74.17 & IV & 69.17 & VI & 58.33 & $\mathrm{X}$ & 72.30 & $\mathrm{~V}$ & 66.25 & VI & $3.53 *$ \\
\hline 5 & Kisan Seva Kendra & 82.92 & II & 80.67 & II & 81.70 & II & 80.83 & II & 82.31 & II & 80.75 & II & $3.95^{*}$ \\
\hline 6 & Literature & 59.58 & $\begin{array}{c}\text { VII } \\
\text { I }\end{array}$ & 69.17 & VI & 75.83 & IV & 60.83 & IX & 67.71 & VII & 65.00 & VII & $3.17 *$ \\
\hline 7 & Group discussion & 78.75 & III & 61.67 & $\begin{array}{c}\text { VII } \\
\text { I }\end{array}$ & 79.17 & III & 78.25 & III & 78.96 & III & 69.96 & $\mathrm{~V}$ & $2.44 *$ \\
\hline 8 & Group meeting & 66.70 & VII & 50.80 & $\mathrm{x}$ & 61.67 & $\mathrm{x}$ & 64.20 & $\begin{array}{c}\mathrm{VII} \\
\mathrm{I} \\
\end{array}$ & 64.19 & $\begin{array}{c}\text { VII } \\
\text { I }\end{array}$ & 57.50 & $\mathrm{X}$ & $2.60^{*}$ \\
\hline 9 & Field day & 54.17 & IX & 56.25 & IX & 63.33 & IX & 71.67 & VI & 58.75 & IX & 63.96 & $\begin{array}{c}\text { VII } \\
\text { I }\end{array}$ & $2.54 *$ \\
\hline 10 & Field visit & 51.25 & $\mathrm{X}$ & 45.80 & $\mathrm{XI}$ & 58.30 & $\mathrm{XI}$ & 69.17 & VII & 54.78 & $\mathrm{XI}$ & 57.49 & $\mathrm{XI}$ & $2.11^{*}$ \\
\hline 11 & Education tour & 49.17 & $\mathrm{XI}$ & 66.67 & VII & 65.83 & $\begin{array}{c}\text { VII } \\
\text { I }\end{array}$ & 55.83 & $\mathrm{XI}$ & 57.50 & $\mathrm{x}$ & 61.25 & IX & 1.67 \\
\hline 12 & Work shop/ seminars & 47.92 & XII & 47.67 & XII & 56.70 & XII & 53.25 & XII & 52.31 & XII & 50.59 & XII & $2.91 *$ \\
\hline
\end{tabular}

$*$ = significant at 5 per cent level of significance 
Table.5 Extent of use of different impersonal cosmopolite channels of agriculture information by the aonla growers

\begin{tabular}{|c|c|c|c|c|c|c|c|c|c|c|c|c|c|c|}
\hline \multirow[t]{3}{*}{$\begin{array}{l}\text { S. } \\
\text { No. }\end{array}$} & \multirow[t]{3}{*}{$\begin{array}{c}\text { Impersonal Cosmopolite } \\
\text { Channel }\end{array}$} & \multicolumn{4}{|c|}{ Jaipur district (N=160) } & \multicolumn{4}{|c|}{ Ajmer district $(\mathrm{N}=80)$} & \multicolumn{4}{|c|}{$\begin{array}{c}\text { Total respondents } \\
(\mathrm{N}=\mathbf{2 4 0})\end{array}$} & \multirow[t]{3}{*}{$\begin{array}{l}\text { Wilcoxen } \\
\text { (Z value) }\end{array}$} \\
\hline & & \multicolumn{2}{|c|}{$\begin{array}{c}\text { Farmers } \\
(\mathbf{n}=\mathbf{8 0})\end{array}$} & \multicolumn{2}{|c|}{$\begin{array}{c}\text { Farm women } \\
(n=80)\end{array}$} & \multicolumn{2}{|c|}{$\begin{array}{c}\text { Farmers } \\
(n=40)\end{array}$} & \multicolumn{2}{|c|}{$\begin{array}{c}\text { Farm women } \\
(\mathrm{n}=40)\end{array}$} & \multicolumn{2}{|c|}{$\begin{array}{c}\text { Farmers } \\
(n=120)\end{array}$} & \multicolumn{2}{|c|}{$\begin{array}{c}\text { Farm women } \\
(\mathrm{n}=120)\end{array}$} & \\
\hline & & MPS & $\begin{array}{l}\text { Ra } \\
\text { nk }\end{array}$ & MPS & $\begin{array}{l}\text { Ra } \\
\text { nk }\end{array}$ & MPS & $\begin{array}{l}\text { Ra } \\
\text { nk }\end{array}$ & MPS & $\begin{array}{l}\text { Ra } \\
\text { nk }\end{array}$ & MPS & $\begin{array}{l}\text { Ra } \\
\text { nk }\end{array}$ & MPS & $\begin{array}{l}\text { Ra } \\
\text { nk }\end{array}$ & \\
\hline 1. & Radio & 84.17 & II & 82.92 & II & 80.83 & II & 78.83 & II & 82.50 & II & 80.88 & II & $3.43 *$ \\
\hline 2. & TV./Film show & 86.25 & I & 85.42 & I & 85.83 & I & 83.30 & I & 86.04 & I & 84.36 & I & 1.87 \\
\hline 3. & News paper & 72.50 & IV & 71.70 & III & 72.50 & IV & 67.50 & $\begin{array}{c}\text { VII } \\
\text { I }\end{array}$ & 72.50 & IV & 69.60 & $\mathrm{~V}$ & $3.82 *$ \\
\hline 4 & Farm Journals /Magazines & 69.58 & $\mathrm{~V}$ & 58.33 & IX & 66.67 & $\mathrm{~V}$ & 61.70 & IX & 68.13 & VI & 60.02 & IX & $3.11 *$ \\
\hline 5 & $\begin{array}{l}\text { Traditional media (Puppet, } \\
\text { local song, drama) }\end{array}$ & 58.33 & $\begin{array}{c}\text { VII } \\
\text { I }\end{array}$ & 60.42 & $\begin{array}{c}\text { VII } \\
\text { I }\end{array}$ & 64.17 & $\begin{array}{c}\text { VII } \\
\text { I }\end{array}$ & 72.50 & $\mathrm{~V}$ & 61.25 & IX & 66.46 & VI & $3.08 *$ \\
\hline 6 & Exhibitions & 59.58 & VII & 63.33 & VI & 64.17 & IX & 69.17 & VI & 61.88 & VII & 66.25 & VII & $2.85 *$ \\
\hline 7 & E-mail/Internet & 53.80 & $\mathrm{X}$ & 55.40 & $\mathrm{X}$ & 62.50 & $\mathrm{X}$ & 56.67 & $\mathrm{X}$ & 58.15 & $\mathrm{X}$ & 56.04 & $\mathrm{X}$ & 1.81 \\
\hline 8 & Poster/ Chart/Circulars & 67.92 & VI & 62.50 & VII & 70.83 & VI & 64.17 & $\begin{array}{c}\text { VII } \\
\text { II }\end{array}$ & 69.38 & $\mathrm{~V}$ & 63.34 & $\begin{array}{c}\text { VII } \\
\text { I }\end{array}$ & $1.98 *$ \\
\hline 9 & Telephone/Mobile Phone & 77.50 & III & 70.80 & IV & 77.50 & III & 77.50 & III & 77.50 & III & 74.15 & III & $2.04 *$ \\
\hline 10 & Youth club /Mahila mandal & 54.17 & IX & 68.33 & $\mathrm{~V}$ & 69.33 & $\begin{array}{c}\text { VII } \\
\text { I } \\
\end{array}$ & 74.17 & IV & 61.75 & $\begin{array}{c}\text { VII } \\
\text { I } \\
\end{array}$ & 71.25 & IV & $2.32 *$ \\
\hline
\end{tabular}

$*=$ significant at 5 per cent level of significance

The accessibility of certain sources of agriculture information has its bearing on the extent of its use by aonla growers. So, the "agriculture supervisor" being easily accessible to the aonla growers might have been contacted regularly with aonla growers in the area for seeking information on improved aonla cultivation practices. Hence, it is suggested that the authorities should be concerned with filling existing vacant posts of agriculture supervisor for the well-being of aonla growers living in the area.

Extent of use of different personal cosmopolite channels of agriculture information by the aonla growing farmers and farm women

The data related with extent of use of different personal cosmopolite channels of agriculture information of the aonla growing farmers and farm women incorporated in Table 4 shows that calculated Wilcoxen ' $Z$ ' value for the extent of use of personal cosmopolite channels viz. 'training, farmers fair (Kisan Mela)', 'result demonstration', 'method demonstration', 'Kisan Seva Kendra', 'literature', 'group discussion', 'group meeting', 'field day', 'field visit' and 'work shop/ seminars' were more than the tabulated value at 5 per cent level of significance. Hence, the null hypothesis was rejected and alternate hypothesis was accepted, which leads to the conclusion that there is a significant difference between aonla growing farmers and farm women with respect to the extent of use of these personal cosmopolite channels of information. Whereas the calculated Wilcoxen ' $Z$ ' value for the 'education tour' was less than the tabulated value at 5 per cent level of significance. Hence, the null hypothesis was accepted and alternate hypothesis was rejected, which leads to the conclusion that there is no significant difference between aonla growing farmers and farm women with respect to the extent of use of these personal cosmopolite channels of information.

It is also apparent from the Table 4 that for aonla growing farmers and farm women "farmer's fair" (MPS 85.02 and 83.75 respectively) and "Kisan Seva Kendra" (MPS 82.31 and 80.75 respectively) were the major personal cosmopolite channels of agriculture information used by majority of the aonla growers and accorded first and second ranks respectively. It also revealed that for aonla growing farmers and farm women "work shop/ 
seminar" (MPS 52.31 and 50.59 respectively) was the least preferred information channel.

In case of aonla growing farmers and farm women "farmer's fair" (MPS 85.83 and 84.17 respectively) and "Kisan Seva Kendra" (MPS 82.92 and 80.67 respectively) in Jaipur district were the major personal cosmopolite channels of agriculture information used by majority of the aonla growers and accorded first and second ranks respectively. It was also revealed that for aonla growing farmers and farm women "work shop/ seminar" (MPS 47.92 and 47.67 respectively) was the least preferred information channel. Whereas, for the aonla growing farmers and farm women in Ajmer district "farmer's fair" (MPS 84.20 and 83.33 respectively) and "Kisan Seva Kendra" (MPS 81.70 and 80.83 respectively) were the major personal cosmopolite channels of agriculture information used by majority of the aonla growers and accorded first and second ranks respectively. It also revealed that for aonla growing farmers and farm women "work shop/ seminar" (MPS 56.70 and 53.25 respectively) was the least preferred information channel as perceived by the respondents.

On the basis of data, it is concluded that 'farmer's fair', 'Kisan Seva Kendra, 'training' and 'result demonstration' were the most important personal cosmopolite channels of agriculture information.

\section{Extent of use of different impersonal cosmopolite channels of agriculture information by the aonla growing farmers and farm women}

The data related with extent of use of different impersonal cosmopolite channels of agriculture information of the aonla growing farmers and farm women incorporated in Table 5 shows that calculated Wilcoxen ' $Z$ ' value for the extent of use of impersonal cosmopolite channels viz. radio, TV/ Film shows, newspapers, farm journals /magazines, traditional media (Puppet, local song, drama), exhibitions, poster/ chart/circulars, telephone/mobile phone and youth club /mahila mandalwere more than the tabulated value at 5 per cent level of significance. Hence, the null hypothesis was rejected and alternate hypothesis was accepted, which leads to the conclusion that there is a significant difference between aonla growing farmers and farm women with respect to extent of use of different these impersonal cosmopolite channels of information. Whereas the calculated Wilcoxen ' $Z$ ' value for the 'e-mail/Internet' was less than the tabulated value at 5 per cent level of significance. Hence, the null hypothesis was accepted and alternate hypothesis was rejected, which leads to the conclusion that there is no significant difference between aonla growing farmers and farm women with respect to the extent of use of this impersonal cosmopolite channel of information.

It is clear from the data in Table 5 that for aonla growing farmers and farm women "TV/Film show" (MPS 86.04 and 84.36 respectively) and "radio" (MPS 82.50 and 80.88 respectively) were the major impersonal cosmopolite channels of agriculture information used by majority of the aonla growers and accorded first and second ranks respectively. It also revealed that for aonla growing farmers and farm women "E-mail/ internet" (MPS 58.15 and 56.04 respectively) was the least preferred information channel.

In case of aonla growing farmers and farm women "TV/Film show" (MPS 86.25 and 85.42 respectively) and "radio" (MPS 84.17 and 82.92 respectively) in Jaipur district were the major impersonal cosmopolite channels of agriculture information used by majority of the aonla growers and accorded first and second ranks respectively.

It also revealed that aonla growing farmers and farm women "E-mail/ internet" (MPS 53.80 and 55.40 respectively) was the least preferred information source.

Whereas the aonla growing farmers and farm women in Ajmer district "TV/Film show" (MPS 85.83 and 83.30 respectively) and "radio" (MPS 
80.83 and 78.83 respectively) were the major impersonal cosmopolite channels of agriculture information used by majority of the aonla growers and accorded first and second ranks respectively.

It also revealed that aonla growing farmers and farm women "E-mail/ internet" (MPS 62.50 and 56.67 respectively) was the least preferred information.

In other words, it was concluded that 'TV/Film show', 'radio' and 'telephone/ mobile phone' were the most impersonal cosmopolite channels of agriculture information as perceived by the aonla growers in the study area.

Majority of the aonla growing farmers and farm women (56.00 per cent and 52.00 per cent respectively) were having high extent of use of agriculture information sources and channels further, a highly significant agreement between the aonla growing farmers and farm women was noticed with regard to their extent of use of agriculture information sources and channels.

The personal localite sources and the impersonal cosmopolite channels of agriculture information were perceived as most extent of use of agriculture information.

\section{Recommendations}

It was also observed that 'agriculture graduate', 'seminar/ workshop', 'agriculture research scientist' and 'e-mail/internet' were perceived as the least used sources and channels of agriculture information by all the categories of aonla growers. Therefore, remedial action should be taken to the use of these sources and channels of agriculture information.
It was found that all the categories of aonla growers used to 'family members', 'progressive farmers', 'friends' and 'neighbours' as their major personal localite sources of agricultural information, these sources were also perceived as the most extent of use personal localite sources. Sometimes, friends and neighbours generally not are competent with technical and practical aspects of improved aonla cultivation practices.

\section{References}

Dhayal, B. L. (2006). "Communication behaviour of ber growers in Chomu Tehsil of Jaipur District of Rajasthan". M.Sc. Thesis RAU, Bikaner, CampusJobner.

Dhayal, B. L., Khan, I.M. and Jangid, M. K. (2012).'Information seeking information of ber growers in Jaipur District of Rajasthan." Rajasthan Journal of Extension Education, vol. 20, pp. 57-60

Gupta, S. P., Amardeep and Kushal (2003). "Utilization of information source by farmers under different production system in Uttranchal and Uttar Pradesh," Manage Extension Research Review, vol. 4(2), pp. 70-80.

Yadav, B.S. (2009). "Information Seeking Behaviour of Fenugreek Growers in Jaipur Region of Rajasthan." Ph.D. Thesis, RAU, Bikaner, Campus-Jobner.

Yadav, B.S. and Khan, I.M. (2005). "Extent of utilization of different information sources used by the cauliflower growing farmers of Govindgarh Panchayat Samiti of Jaipur district". $3^{\text {rd }}$ National Extension Education Congress (April 27-29, 2005) held at NDRI, Karnal (Haryana) pp. 136137.

\section{How to cite this article:}

Choudhary, S. and Khan, I.M. 2017. Extent of Use of Different Agriculture Information Sources and Channels by the Aonla Growers. Int.J.Curr.Microbiol.App.Sci. 6(12): 1769-1777. doi: https://doi.org/10.20546/ijcmas.2017.612.200 